Article:

\title{
Phantom membrane microfluidic cross-flow filtration device for the direct optical detection of water pollutants
}

R. Lanfranco, J. Saez, E. Di Nicolò, F. Benito-Lopez, M. Buscaglia

Sensors and Actuators B: Chemical 257 : 924-930 (2018)

This work is made available online in accordance with publisher policies. To see the final version of this work please visit the publisher's website. Access to the published online version may require a subscription.

Link to publisher's version:

http://doi.org/10.1016/j.snb.2017.11.024

Copyright statement: (c) 2017 Elsevier Ltd. Full-text reproduced in accordance with the publisher's self-archiving policy.

This manuscript version is made available under the CC-BY-NC-ND 4.0 license http://creativecommons.org/licenses/by-nc-nd/4.0

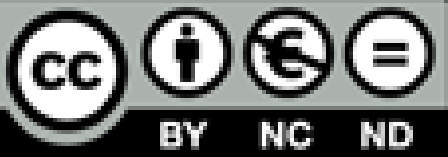




\title{
Phantom Membrane Microfluidic Cross-Flow Filtration Device for the Direct Optical
}

\author{
Detection of Water Pollutants
}

\author{
R. Lanfranco, J. Saez, E. Di Nicolò, F. Benito-Lopez, M. Buscaglia
}

\section{A B S T R A C T}

\begin{abstract}
The diffusion of autonomous sensing platforms capable of a remote large-scale surveillance of environ-mental water basins is currently limited by the cost and complexity of standard analytical methods. In order to create a new generation of water analysis systems suitable for continuous monitoring of a large number of sites, novel technical solutions for fluid handling and detection are needed. Here we present a microfluidic device hosting a perfluorinated microporous membrane with refractive index similar to that of water, which enables the combination of filtration and label-free sensing of adsorbing substances, mainly pollutants, in environmental water samples. The cross-flow design of the microfluidic device avoids the clogging of the membrane due to particulate, whereas molecules with some hydrophobic moiety contained in the crossing flow are partially retained and their adhesion on the inner surface of the membrane yields an increase of light scattering intensity, which can be easily measured using a sim-ple instrument based on Light Emitting Diode illumination. By cycling sample water and pure water as a reference, we demonstrate the detection of $0.5 \square \mathrm{M}$ of a model cationic surfactant and regeneration of the sensing surface. The optical response of the membrane sensor was characterized using a simple theoretical model that enables to quantify the concentration of target molecules from the amplitude and kinetics of the measured binding curves. The device was tested with real water samples containing large amount of environmental particles, without showing clogging of the membrane, and enabling nonspecific quantification of adsorbing substances in a few minutes.
\end{abstract}

\section{Introduction}

Contamination of water is a constant concern all over the world. The availability of uncontaminated water for drinking and for the agricultural processes is a fundamental need of any human being. Moreover, pollution of water basins has a strong impact on the overall environmental equilibrium. Every day, as a result of both domestic and industrial activities, substances threatening the survival of flora and fauna are poured in the aquatic ecosystems. Some of the most widespread contaminations include faecal pollutants [1], harmful organic and inorganic substances, oils and emulsi- fiers [2,3]. Among these, hydrophobic and amphiphilic compounds tend to migrate to the air/water interfaces, preventing proper oxygen exchange, and to accumulate in many living organisms, hence threatening their life [4]. Therefore, it would be extremely important to develop analytical systems capable of continuous and extensive monitoring of molecular pollutants in water down to the micromolar range. In analytical laboratories, liquid chromatography and mass-spectroscopy are widely employed approaches to detect contaminants in liquids and solubilized media [5-7]. However, they require several processing steps and highly specialized instrumentation and hence they are not suitable for the implementation in autonomous platforms capable of on-site, realtime monitoring. Nowadays, the proper quantification of most contaminants still requires the highest analytical performance only obtained in the laboratory by highly trained personnel [8]. Consequently, the required sample collection and transportation necessarily prevents the possibility of a rapid intervention in case of contamination. In order to overcome these limits, different kinds 
of autonomous and deployable analytical platforms have been proposed [9-11]. However, their overall cost per analysis is typically high and consequently the sampling frequency and the number of sampled sites are largely affected by budget restrictions. Despite the constant innovation towards more sensitive and robust detection methods [12], the components of the analytical platform that often have a higher impact on the cost of production and maintenance are still those devoted to sampling and handling of the liquid, such as pumps, valves and filters.

Recently, novel sensing substrates, either planar or surfacestructured, have been proposed for the realization of analytical systems with improved performance for the detection of polluting molecules, possibly to be used outside the laboratories [13-15]. Among these, the use of perfluorinated polymers with refractive index similar to that of water represents a promising approach to fabricate cost-effective, and sensitive label-free biosensors. In fact, the detection and characterization of biomolecular targets in aqueous samples were demonstrated with perfluorinated materials with different shapes, including planar surfaces, nano-particles and microporous membranes [16-19]. In particular, in the Reflective Phantom Interface (RPI) method, the intensity of light reflected by a planar interface between Hyflon $\mathrm{AD}^{\circledR}$ (Solvay Specialty Polymer, Italy) and an aqueous sample enables to quantify in real-time the binding of biomarkers to immobilized antibodies without the need of labeling agents such as fluorescent or colorimetric moieties. Similarly, in the Scattering Phantom Interface (SPI) approach, the adhesion of proteins or surfactants on the surface of dispersed nanoparticles or in porous membranes yields to an increase of the intensity of the scattered light. The optical response of these different materials has been modelled in detail [19]. Although the highest theoretical sensitivities can be obtained using the dispersions of nanoparticles, their possible aggregation makes this system suitable only for molecular targets and sample solutions that do not destabilize the suspension. In contrast, this limitation does not apply to planar surfaces or to microporous membranes. For those applications in which a reduced volume of sample is not a requirement (e.g. water monitoring), a membrane sensor is preferable, especially if its filtering capability can be also exploited.

In the quest for novel paradigms for environmental analysis enabling rapid detection and identification of compounds at the point of need, microfluidic devices are emerging as versatile tools offering many advantages, including the possibility of cost effective automation, low reagent consumption and multiplexing $[20,21]$. Different materials for the fabrication of microfluidic devices have been proposed since the beginning of the investigations in microfluidic technology [22]. In particular, COP is being increasingly used because it shows outstanding properties while being cheap and optically transparent $[23,24]$. Moreover, the realization of hybrid devices combining microfluidics and innovative sensing substrates represents a particularly promising approach to design new concepts of autonomous analytical systems with reduced complexity $[25,26]$.

In this work, we present the fabrication and characterization of a hybrid microfluidic device based on COP and embedding a novel perfluorinated microporous membrane isorefractive to water that enables label-free optical sensing of adsorbing substances in aqueous samples. The device exploits the SPI detection method and yields an increase of the intensity of scattered light upon adhesion of molecular compounds onto the inner surface of the membrane. The amplitude and the time dependence of the response for different concentrations of a model cationic surfactant are shown to scale according to a simple pseudo-first order interaction model. The affinity and kinetic constants extracted from the analysis are ascribed to the intrinsic interactions between the perfluorinated interface and the hydrophobic moiety of the compound. The membrane configuration in the microfluidic device exploits a cross-flow geometry that enables to avoid the clogging of the membrane pores in presence of particulate in the sample. The microfluidic sensor allows for continuous water monitoring by alternating the flow of sample with clean water as reference. The detection of about $0.5 \mu \mathrm{M}$ of surfactant is achieved by this approach with multiple cycles of about $30 \mathrm{~min}$ each at a flow rate of $150 \mu \mathrm{L} \mathrm{min}^{-1}$. The performance of the sensor was tested with real river water samples collected from two different sites, upstream and downstream an urban area. As expected, the optical response of the latter sample indicates a higher level of pollution, compatible with a four-fold increase of concentration. Moreover, these tests demonstrate that the cross-flow design of the microfluidic device enables an efficient sampling at high flow rates without any kind of pre-filtration step, avoiding the clogging of the membrane even when the sample contains high levels of particulate, as commonly found in river water.

\section{Materials and methods}

\subsection{Membrane sensor fabrication and characterization}

We fabricated a microporous membrane made of Hyflon AD $40^{\circledR}$, a copolymer of Tetrafluoroethylene (TFE) and $40 \%$ of 1,1,2, trifluoro5-trifluoromethoxy-1,3 dioxole (TTD) [27]. This material has a fully amorphous structure and a refractive index similar to that of water. The membrane was realized by non-solvent induced phase separation (NIPS) technique [28], as described in SI, and was characterized by different analytical methods. The morphology was investigated by Scanning Electron Microscopy (SEM) (Fig. 1a). The thickness measured by a micrometer is $90 \mu \mathrm{m}( \pm 10 \mu \mathrm{m})$. The porosity (i.e. volume of voids over the total volume of the membrane) obtained by weighing the membrane when soaked with isopropanol and dried is $73 \% \pm 1 \%$. The amount of residual solvent was estimated as $1.1 \%$ by Thermal Gravimetric Analysis (TGA). The mean pore diameter of $9.1 \mu \mathrm{m}$ was obtained by liquid-gas displacement method using a capillary flow porometer according to ASTM F316. The membrane refractive index $n_{m}=1.3285$ was measured from the dependence of the scattered light intensity on the refractive index of water-glycerol solutions. The hydrophobicity of Hyflon AD $40^{\circledR}$ is slightly smaller than that of fully fluorinated polymers such as PTFE, because of the presence of a partially hydrogenated comonomer that completely prevents crystallization and gives rise to a fully amorphous structure. The contact angle for water of a planar surface of Hyflon $A D^{\circledR}$ is in the range $110^{\circ}-120^{\circ}$. Therefore, efficient wetting of the microporous membrane with water was achieved by pre-wetting with a 30\%-70\% vol ethanol-water solution.

\subsection{Microfluidic device design and fabrication}

The membrane is hosted in a hybrid microfluidic device in a cross-flow configuration to avoid the clogging in presence of particulate. A schematic representation of the microfluidic design is shown in Fig. 1b and c. The device was fabricated with COP and pressure sensitive adhesive (PSA) using a multilayer technique as explained before [24], and the membrane was positioned during the assembly. The microfluidic device has two inlets ( 1 and 2 ) that merge in a single channel tangential to the membrane and directed to the outlet 1 . Below the membrane, a perpendicular channel brings the filtered sample towards the outlet 2 . The perfluorinated membrane is suspended in a rectangular space with size $5 \times 3 \mathrm{~mm}$ placed at the intersection of the two channels of the device (tangential and perpendicular channels). The lengths and cross sections of the channels are designed in order to balance the hydrodynamic resistance of the membrane and to obtain similar flow values in both outlets. 

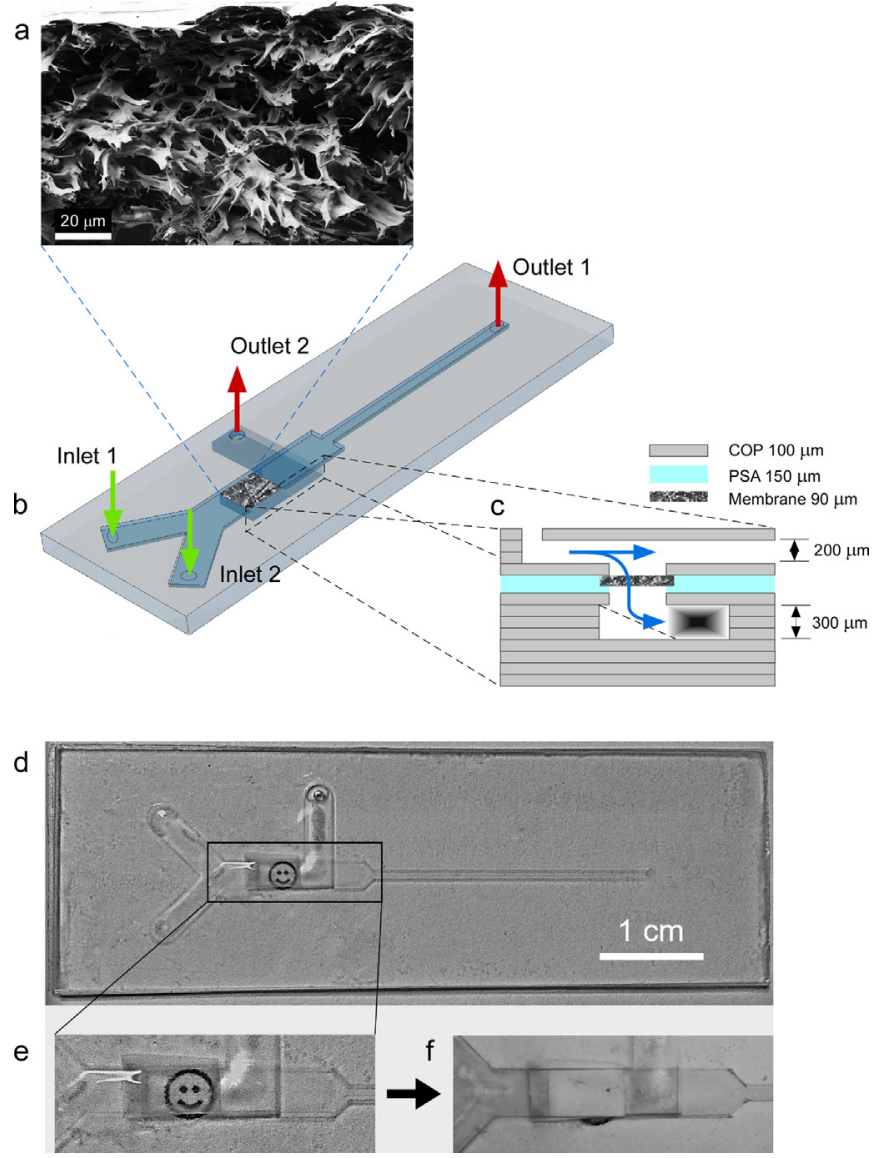

Fig. 1. Scheme and picture of the microfluidic device hosting the microporous membrane. a) SEM image of the microporous membrane. b) 3D view of the cross-flow geometry that enables the simultaneous filtration process and optical detection. c) Scheme of the cross-section of the microfluidic device. d) Image of the fabricated device. e) Enlarged view of the part hosting the membrane soaked with water and f) dried.

The microfluidic device was fabricated by rapid prototyping using the FC8000-60 cutting plotter (Graphtec ${ }^{\circledR}$, USA) for the cutting of the COP and PSA layers. The COP layers of $100 \mu \mathrm{m}$ thickness (Zeonor COP sheets purchased from Zeonex, Germany) were xurographied and bonded by thermocompression. In order to deposit the membrane within the microfluidic channel in a robust way, the top and the bottom layers of the microfluidic device were bonded together with a PSA layer of $150 \mu \mathrm{m}$ (ArCare ${ }^{\circledR} 8939$ PSA, Adhesive Research, Ireland) that acts as a substrate to hold the membrane (Fig. 1c). The final hybrid microfluidics are composed by 12 layers of COP with a total section of $7.5 \times 2.5 \mathrm{~mm}$, and $1.4 \mathrm{~mm}$ total thickness $[29,30]$. The channel that passes above the membrane has a height of $200 \mu \mathrm{m}$ and a width $1 \mathrm{~mm}$. The perpendicular channel, which passes below the membrane, has a height of $300 \mu \mathrm{m}$ and a width of $3 \mathrm{~mm}$.

The perfluorinated membrane was embedded into the microfluidic device during the assembly. Before this step, the membrane was soaked for $24 \mathrm{~h}$ with MilliQ water at room temperature and then cut using a scalpel. The device was finally filled with water and degassed for $40 \mathrm{~min}$ at controlled temperature $\left(30^{\circ} \mathrm{C}\right)$ to eliminate trapped air bubbles. Female luer connectors were glued to the input channels using a double side PSA layer with the diameter of the luer. Home-made male connectors that minimize dead-volumes were used directly connected to a syringe pump (RS 232-Genie Touch Kent Scientific Corporation) using a $200 \mathrm{~mm}$ long silicone tube with diameter $100 \mu \mathrm{m}$.

\subsection{Optical setup and measurement}

A custom optical apparatus to measure the intensity of light scattered by the membrane was designed and realized on an optical board using optomechanical components purchased from Thorlabs (Fig. S1). The light of a LED with peak wavelength $\lambda=592 \mathrm{~nm}$ (Luxeon Rebel Amber) was collimated and shaped in order to reduce the stray light contribution possibly originated from the edges of the channels or the membrane. A lens collects the back-scattered light at about $135^{\circ}$ relative to the transmitted beam and forms an image of the membrane surface on a CCD camera (Stingray Allied Technology, Germany) to monitor the scattered light intensity. The microfluidic device and the optical system are placed in a black enclosure to prevent spurious signals due to ambient light. The measurements are performed at room temperature without any temperature control system. A LabView program registers the images captured by the CCD camera at a speed of $1 \mathrm{fps}$. The images are analyzed using ImageJ: the contrast is adjusted in the same way for all the data collected and a moving average is performed over 5 frames to reduce the noise. Then, the averaged intensity of the image acquired from the membrane area is computed as a function of time.

The intensity of light scattered by the membrane and acquired by this optical setup provides a quantification of molecular adsorption on the inner surface of the membrane. Experiments were performed using the cationic surfactant benzyldimethylstearylammonium chloride monohydrate (SBSAC) purchased from Sigma-Aldrich. The surfactant was dissolved either in deionized water or in a sample of water collected from Lambro river at Ponte Albiate (site 1) and Melegnano (site 2) in Italy. Neither centrifugation nor filtration steps were performed before the measurement. All the experiments were performed at room temperature $\left(25^{\circ} \mathrm{C}\right)$. After each experiment, the tubes and connectors of the fluidic system were washed with fresh water and ethanol. The microfluidic device hosting the membrane was washed by flowing for at least an hour a 30\%-70\% vol ethanol-water solution after each set of measurements. With this procedure, a single microfluidic device could be used for more than a dozen sets of measurements with no signs of degradation.

\subsection{Optical model}

The peculiar optical properties of the microporous membranes of Hyflon $A D^{\circledR}$ are due to the refractive index similar to that of water and to the amorphous structure of the material. When dried, the membrane appears white because of the refractive index mismatches with air that yields to a large fraction of scattered light. When soaked with an aqueous solution, the membrane becomes highly transparent (Fig. 1e) because of the small difference of refractive index between the solid and the liquid phases and the absence of microcrystalline domains. In this condition, the adsorption of molecular compounds with higher refractive index - as basically any carbon-based compound - on the inner surface of the membrane induces an increase of the scattered light by SPI that can be easily detected by a simple optical system [19]. An optical model that describes the increment of scattered light intensity due to molecular adsorption on an index-matched porous material was previously derived by scattering models of spheres coated by a thin shell with different refractive index [31,32]. Remarkably, for pore radius bigger than about $100 \mathrm{~nm}$, the model for the scattered light intensity is formally identical to that for the light reflected by a thin layer between two media with similar refractive indices, as derived 


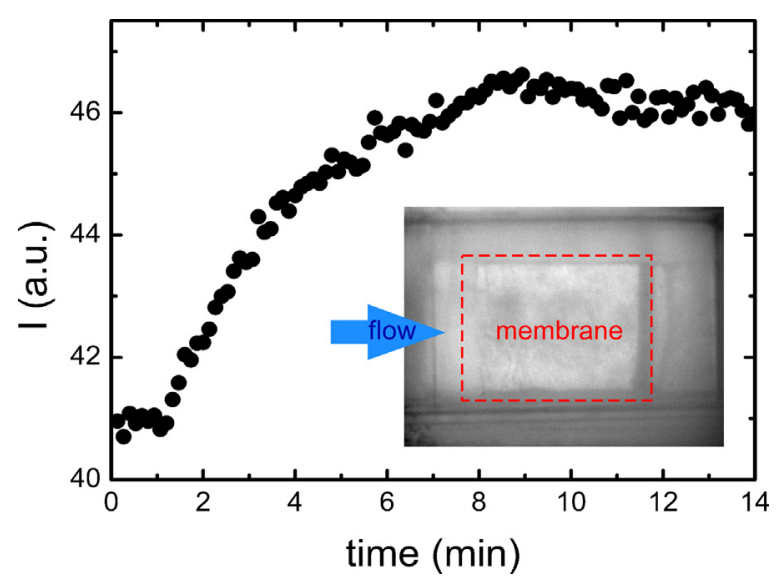

Fig. 2. Intensity of light scattered by the membrane upon adsorption of surfactant. The average brightness of the pixels corresponding to the membrane image increases during the flow of $4 \mu \mathrm{M}$ SBSAC surfactant, indicating an increase of scattered light intensity. Inset: image of the membrane hosted in the microfluidic device acquired by the optical set-up. The image brightness has been enhanced for clarity purposes.

by Fresnel equations $[16,19]$. The intensity $I$ of light scattered (or reflected) by a thin layer of thickness $h$ is given by:

$\frac{I}{I_{0}}=1+\left(\frac{h}{h^{*}}\right)^{2}$

where $I_{0}$ is the scattered light intensity in the absence of the layer and $h^{*}$ corresponds to the layer thickness yielding to $I=2 I_{0}$. Eq. (1) enables to convert the measured intensity of scattered light into the dimensionless parameter $h / h^{*}$, which represents the amount of molecules adsorbed on the inner surface of the membrane. An absolute quantification of $h$ is obtained through an estimate of the value of $h^{*}$, which depends on the refractive indices of the membrane $\left(n_{m}\right)$, of the solution $\left(n_{s}\right)$ and of the adsorbed molecular layer $\left(n_{l}\right)$ by $[19]$ :

$h^{*}=\left|\frac{n_{m}^{2}-n_{s}^{2}}{n_{l}^{2}-n_{s}^{2}}\right| \frac{1}{k}$

where $k=4 \pi n_{s} \sin \left(\theta_{s} / 2\right) / \lambda$ is the scattering vector and $\theta_{s}$ is the angle between the incident and the scattered rays. For the materials and the optical setup used in this study $h^{*} \approx 0.45 \mathrm{~nm}$. In order to analyze the experimental data, a background contribution due to stray-light $I_{f}$ is subtracted from the measured intensity to obtain $I$, which then is converted into $h$ through Eq. (1).

\section{Results and discussion}

\subsection{Optical response to surfactant adsorption}

The microporous membrane sensor embedded in the microfluidic device yields a label-free SPI optical signal upon adsorption of molecules present in the sample solution. The membrane material combines a high optical transparency in water with the hydrophobicity typical of perfluoropolymers. Therefore, compounds with a hydrophobic moiety, such as surfactants, are retained on the inner surface of the membrane and the formation of a thin layer with refractive index higher than the membrane material and the solution induces an increase of scattered light intensity according to Eq. (1). The microfluidic design provides a wide optical access to the area of the membrane, hence enabling to illuminate its surface and to collect the scattered light from a large angular range using an imaging system. Fig. 2 reports an image of the membrane hosted in the microfluidic device acquired by the CCD camera during the measurement. As shown in Fig. 2, upon the flow of $4 \mu \mathrm{M}$
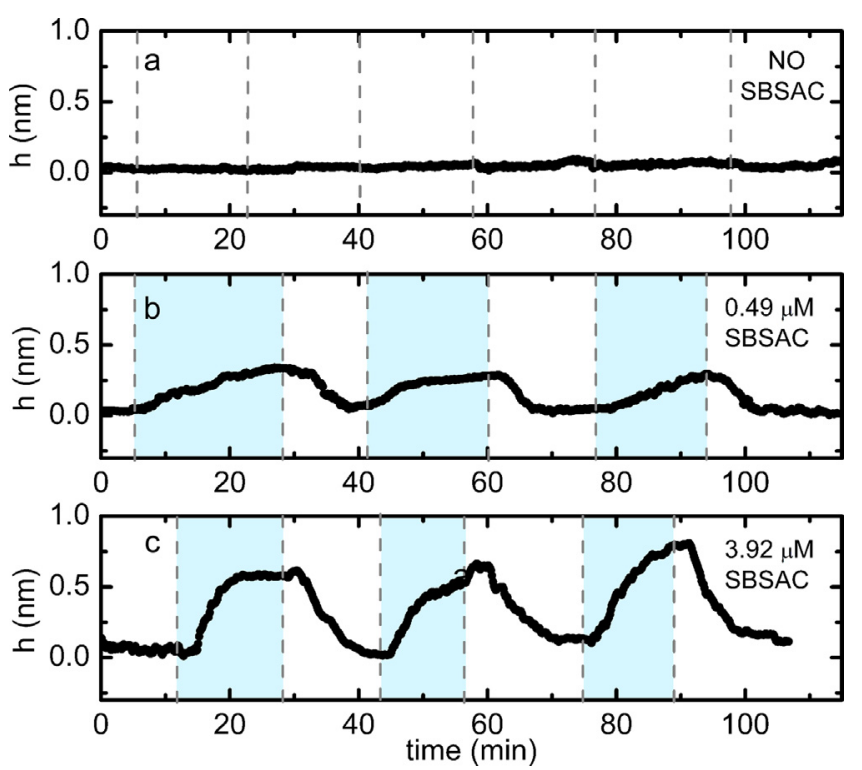

Fig. 3. Surfactants detection and regeneration of the device. Subsequent injection of surfactant (light blue) and water (white) were performed at the flow rate of $150 \mu \mathrm{L} \mathrm{min}^{-1}$. a) Injections of pure water representing the baseline signal. b) Injection of $0.49 \mu \mathrm{M}$ of SBSAC and c) $3.92 \mu \mathrm{M}$ of SBSAC alternated to pure water. The highest concentration of surfactant yields a higher and more rapid increase of the adsorbed layer thickness. (For interpretation of the references to colour in this figure legend, the reader is referred to the web version of this article.)

SBSAC surfactant, the average value of the pixels corresponding to the membrane image becomes larger, indicating an increase of the intensity of light scattered by the membrane. The subsequent washing with pure water enables the desorption of the molecules from the membrane and induces a decrease of scattered light intensity until the initial condition is recovered. The signal obtained with clean water represents a reference and the quantification of surfactant concentration is achieved from the rate of increase of the scattered light intensity upon switching to the sample flow.

The effective thickness $h$ of the adsorbed layer obtained by cycling sample and reference flow is reported in Fig. 3. The flow rate through the membrane was maintained at $150 \mu \mathrm{Lmin}^{-1}$ to enable a rapid response time upon the injection of sample. Fig. 3a shows that, if the sample solution does not contain surfactants, the optical signal measured from the membrane does not change upon alternating the flows, as expected. In contrast, a concentration as small as $0.5 \mu \mathrm{M}$ of cationic surfactant SBSAC induces a clear modulation of the light scattering signal (Fig. 3b). The intensity of scattered light increases almost linearly in correspondence of the sample flow and decreases with pure water, indicating that surfactants are desorbing from the inner surface of the membrane. An observation time of about $10-20 \mathrm{~min}$ is sufficient to clearly detect the signal associated to each switch of sample solution. With a higher concentration of surfactant of about $4 \mu \mathrm{M}$, the measured signal increases more rapidly and tends to reach a larger asymptotic value of $h$ (Fig. 3c). Alternated flows of sample solution and pure water yield to a cyclic oscillation of the optical signal, whose average amplitude and response time depend on the concentration of adsorbing molecules in the water sample. Therefore, the membrane embedded into the microfluidic device acts as optical label-free SPI sensor making directly visible both molecular adsorption and desorption. The relative standard deviation of the amplitude $h$ for a single cycle is about $25 \%$ but the accuracy can be increased through repeated cycles of adsorption and desorption.

The effective thickness $h$ of the molecular layer represents a quantification of the amount of adsorbed molecules. A simple approach to interpret the amplitude and kinetics of this quan- 

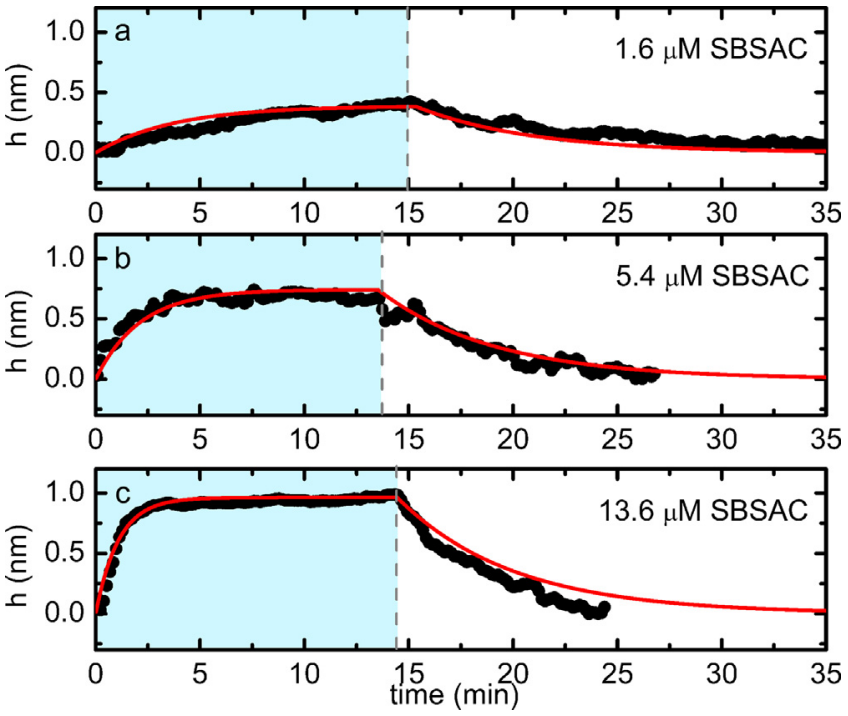

Fig. 4. Characterization of adsorption parameters. Adsorption and desorption of surfactant were studied using a pseudo-first order kinetic model. Increasing concentrations of surfactant (light blue), $1.6 \square \mathrm{m}$ (a), $5.4 \square \mathrm{m}$ (b), $13.6 \square \mathrm{m}$ (c), were injected in the device, and followed by a washing step with water (white). The red curves represent the fits with the exponential functions obtained by the model. (For inter-pretation of the references to colour in this figure legend, the reader is referred to the web version of this article.)

tity upon changes of surfactant concentration $c$ in solution can be derived by the Langmuir model for adsorption [33]. Accordingly, the adsorption curves display exponential growth behaviour as a function of time $t[16]$ :

$\mathrm{h}=\mathrm{h}_{\mathrm{eq}} 1-\mathrm{e}^{-\Gamma \mathrm{t}}$

The asymptotic value $h_{\text {eq }}$ depends on the concentration $c$ and on the equilibrium constant for desorption $\mathrm{K}_{\mathrm{d}}$ as

$\mathrm{h}_{\mathrm{eq}}=\frac{\mathrm{h}_{0}}{1+\underset{\mathrm{c}}{\mathrm{K}}}$

where $h_{0}$ is the plateau value of $h$ at high concentrations. The rate $\Gamma$ of the exponential growth depends on the observed kinetic rate constants for adsorption and desorption, $\mathrm{k}_{\text {on }}$ and $\mathrm{k}_{\text {off }}$, respectively, as

$\Gamma=\mathrm{k}_{\mathrm{on}} \mathrm{c}+\mathrm{k}_{\mathrm{off}}$

where $k_{\text {off }} / k_{\text {on }}=K_{d}$. The values of $k_{\text {on }}$ and $k_{\text {off }}$ depend on the intrin-sic kinetic rates of the molecule-surface interaction as well as on the transport of molecules into the membrane [19]. Fig. 4 shows that the adsorption and desorption curves expressed in terms of the effective thickness $\mathrm{h}$ of the adsorbed molecular layer scale with

the surfactant concentration $c$ according to this model. For $c<\mathrm{K}_{\mathrm{d}}$ the

exponential curves for adsorption and desorption have a simi-

lar rate given by $\Gamma \approx \mathrm{k}_{\text {off }}$. For $c>\mathrm{K}_{\mathrm{d}}$ the rate of the adsorption curve increases with $\mathrm{c}$, whereas the desorption rate for a rapid decrease of concentration from $c$ to zero is always $k_{\text {off }}$. The measured rates are coherent with the extracted value of $\mathrm{K}_{\mathrm{d}}$ that determines the asymptotic amplitude of the curve through Eq. (4). From the fit of

these data, the values of $\mathrm{h}_{0}=1.2( \pm 0.3) \mathrm{nm}, \mathrm{K}_{\mathrm{d}}=3( \pm 1) \square \mathrm{M}, \mathrm{k}_{\mathrm{on}}=900$ $( \pm 190) \mathrm{M}^{-1} \mathrm{~s}^{-1}$ and $\mathrm{k}_{\text {off }}=0.003( \pm 0.0006) \mathrm{s}^{-1}$ are extracted, where the uncertainties are obtained from the standard deviations of five measurements. These values can be compared to those obtained studying the adsorption interaction on a prism made of Hyflon $A D^{\circledR}$ immersed in a cuvette [19]. In that case, the extracted desorp-tion constant was about $0.16 \square \mathrm{M}$, indicating a stronger interaction between the surfactant and the prism surface relative to the mem-brane surface, and $h_{0}$ was about two-fold higher, suggesting that

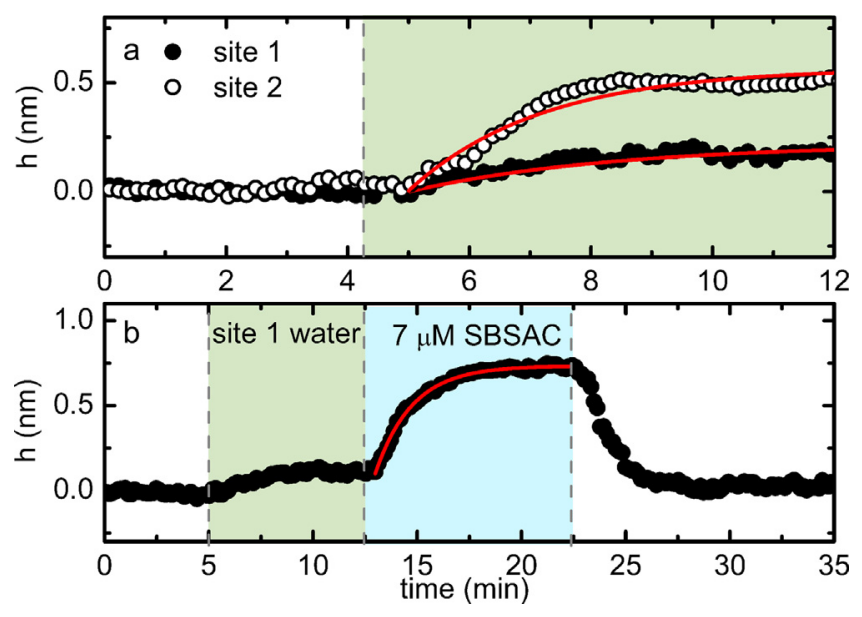

Fig. 5. Surfactant detection in real river water samples. a) Effective thickness $h$ of adsorbed layer obtained with samples of river water (light green) collected from two different locations (site 1 and site 2). The red curves represent the fits using the adsorption parameters extracted from the data of Fig. 4 and with $\mathrm{c}=1 \square \mathrm{M}$ (black dots) and $\mathrm{c}=4 \square \mathrm{M}$ (white dots). b) Comparison of the signal obtained for the sample from site 1 with a sample of $7 \square \mathrm{M}$ of SBSAC. The red curve represents the fit using the adsorption parameters for SBSAC in buffer solution. (For interpretation of the references to colour in this figure legend, the reader is referred to the web version of this article.)

a more complete coverage of the surface was achieved [34]. In fact, the production process of the prism and the membrane are very different. The prism was realized by molding and its surface was mechanically polished, whereas the membrane is produced by non-solvent induced phase separation and its inner surface is only cleaned through repeated washing with ethanol. The higher affinity of the prism suggests a different chemical composition or purity of the interface relative to the membrane. However, despite the higher value of $\mathrm{K}_{\mathrm{d}}$, the membrane investigated here provides a clear signal for surfactant concentrations as low as $0.5 \square \mathrm{M}$. Accord-ingly, higher affinities and hence higher sensitivities are expected to be achievable also with the membrane through the optimization of the production and cleaning processes.

\subsection{Performance of the device with river water samples}

The cross-flow design of the microfluidic device enables the analysis of samples even if they contain particles, because the particles larger than the membrane pores are filtered out by the tangential flow directed to the channel outlet 1 . In order to prove this concept, we tested the device with real river water collected from the Lambro river, nearby the city of Milano. Inspection by optical microscopy showed a high amount of particles with size smaller than $10 \square \mathrm{m}$, corresponding to about $10^{7}$ particles $\mathrm{mL}^{-1}$ (Fig. S2). Despite this, no clogging of the membrane was observed after about an hour of flow at $150 \square \mathrm{L} \mathrm{min}{ }^{-1}$ and the two output chan-nels maintained their flow values during the measurement. The analysis was repeated on water samples collected from two sites of the same river. The results are shown in Fig. 5a. The first site is upstream the city (site 1) and the second is downstream (site 2). The increase of optical signal indicates the formation of an adsorbed molecular layer on the inner surface of the membrane. The sample collected downstream has larger amplitude and faster rate, hence indicating a larger amount of adsorbing molecules, in agreement with the expected increase of pollution after passing the urban area of Milano. Notably, the SPI detection provides a direct and abso-lute quantification of the adsorbed layer thickness through Eq. (1), without the need of a reference sample for calibration. After about $8 \mathrm{~min}$, the effective thickness reached a plateau of about $\mathrm{h}=0.2 \mathrm{~nm}$ and $\mathrm{h}=0.5$ $\mathrm{nm}$ for site 1 and site 2 , respectively. 
Given the non-specific nature of the adsorption interaction, different kinds of substances possibly present in the river water can adhere to the membrane surface, including components of gasoline or biomolecules [19]. Assuming the presence of compounds with interaction parameters similar to that of SBSAC, a qualitative comparison to the surfactant response can be performed. From the analysis of the adsorption curves, the effective thickness of the adsorbed layer and the kinetics would correspond to a concentration of the cationic surfactant of about $1 \mu \mathrm{M}$ and $4 \mu \mathrm{M}$ for site 1 and site 2 , respectively. As a further comparison, Fig. $5 \mathrm{~b}$ shows the signal obtained by the injection of $7 \mu \mathrm{M}$ SBSAC after the injection of the river water sample of site 1 . The larger signal of the cationic surfactant confirms that the signal of the river water samples would correspond to that of lower concentrations of SBSAC. Remarkably, the signal of SBSAC is similar to that obtained at the same concentration using a clean membrane in buffer solution, hence suggesting no degradation of the membrane response with real river samples. Moreover, the subsequent flow of pure water induced the desorption of the molecules (Fig. 5b) and enabled the regeneration of the membrane sensor before another measurement cycle. Overall, these experiments demonstrate the feasibility of using the membrane sensor device to monitor the content of adsorbing substances, mainly pollutants, in river water samples, without the need of preparations steps or reagents.

\section{Conclusions}

We realized and tested a novel label-free optical sensor formed by a perfluorinated microporous membrane embedded in a hybrid microfluidic device. The index matching between the membrane and water enables achieving the SPI conditions and effectively amplifies the optical scattering signal measured upon adhesion of molecules on the inner surface of the membrane. Through an extremely simple optical interrogation, the real-time signal provides a non-specific quantification of adsorbing substances, mainly pollutants, in water samples. The membrane is placed across two channels in order to filter the sample solution in a cross-flow configuration, hence avoiding the clogging of the membrane pores. The system can be easily regenerate by washing with clean water. This enables the continuous monitoring of a water basin with no accumulation of waste products. The device represents a new concept of microfluidic label-free SPI optical sensor with filtering capability, potentially suitable for different kinds of samples containing particulate, including blood and food matrices.

We tested the sensor with a model cationic surfactant and demonstrated the direct detection at different concentrations, down to $0.5 \mu \mathrm{M}$. The amplitude and the response time of the optical signal are consistent with a simple model for binding, by which we estimated the equilibrium and kinetic parameters of the interaction with the sensing surface. According to this model, the whole shape of the binding curve as a function of time is informative in order to estimate the concentration of molecules in unknown samples. This concept was applied to the analysis of real river water collected at two different sites and enabled to quantify the amount of non-specific adsorbing substances. Despite the large amount of particulate, the analysis of river water demonstrated the effectiveness of the adopted microfluidic cross-flow design to avoid the clogging of the membrane. Therefore, this system enables the real-time continuous monitoring of water without pre-treatment of the sample and without the use of reagents. These features are extremely important to develop a new generation of low-cost autonomous platforms for large scale environmental monitoring. Further developments of this sensor are expected to enhance the specificity of detection by functionalizing the inner surface of the membrane with specific bio-receptors (i.e. antibodies) or by adding a spectral analysis of the optical signal without affecting the main features of the system.

\section{Acknowledgements}

This project has received funding from the European Union's Seventh Framework Programme (FP7) for Research, Technological Development and Demonstration through the NAPES project (grant agreement no. 604241). FBL acknowledges the Ramón y Cajal Programme (Ministerio de Economía y Competitividad), Spain. FBL personally acknowledges to Elkartek (KK-2015/00088) Grant from the Gobierno Vasco and funding support from Gobierno de España, Ministerio de Economia y Competitividad, with Grant No. BIO201680417-P and to Marian M. De Pancorbo for letting him to use her laboratory facilities at UPV/EHU. PSA was generously provided by Adhesive Research, Ireland. We thank Aurora Giavazzi for helping in the collection of river water samples.

\section{References}

[1] D.-Y. Lee, H. Lee, J.T. Trevors, S.C. Weir, J.L. Thomas, M. Habash, Characterization of sources and loadings of fecal pollutants using microbial source tracking assays in urban and rural areas of the Grand River Watershed, Southwestern Ontario, Water Res. 53 (2014) 123-131, http://dx.doi.org/10. 1016/j.watres.2014.01.003.

[2] S.D. Richardson, S.Y. Kimura, Water analysis: emerging contaminants and current issues, Anal. Chem. 88 (2016) 546-582, http://dx.doi.org/10.1021/acs. analchem.5b04493.

[3] S.D. Richardson, T.A. Ternes, Water analysis: emerging contaminants and current issues, Anal. Chem. 86 (2014) 2813-2848, http://dx.doi.org/10.1021/ ac500508t.

[4] S. Rebello, A.K. Asok, S. Mundayoor, M.S. Jisha, Surfactants: toxicity, remediation and green surfactants, Environ. Chem. Lett. 12 (2014) 275-287, http://dx.doi.org/10.1007/s10311-014-0466-2.

[5] A.R.S. Ross, X. Liao, A novel method for the rapid determination of polyethoxylated tallow amine surfactants in water and sediment using large volume injection with high performance liquid chromatography and tandem mass spectrometry, Anal. Chim. Acta 889 (2015) 147-155, http://dx.doi.org/ 10.1016/j.aca.2015.06.046.

[6] E. Olkowska, Ż. Polkowska, J. Namieśnik, A solid phase extraction-ion chromatography with conductivity detection procedure for determining cationic surfactants in surface water samples, Talanta 116 (2013) 210-216, http://dx.doi.org/10.1016/j.talanta.2013.04.083.

[7] F. Hernández, M. Ibáñez, T. Portolés, M.I. Cervera, J.V. Sancho, F.J. López, Advancing towards universal screening for organic pollutants in waters, J. Hazard. Mater. 282 (2015) 86-95, http://dx.doi.org/10.1016/j.jhazmat.2014. 08.006.

[8] M. Marchetti, I. Durickovic, Raman spectroscopy as polyvalent alternative for water pollution detection, IET Sci. Meas. Technol. 8 (2014) 122-128, http://dx. doi.org/10.1049/iet-smt.2013.0143.

[9] Y. Zhao, X. Li, Colorimetric and fluorometric detection of anionic surfactants with water soluble sensors, Sens. Actuators B Chem. 209 (2015) 258-264, http://dx.doi.org/10.1016/j.snb.2014.11.122.

[10] C. Xing, L. Liu, S. Song, M. Feng, H. Kuang, C. Xu, Ultrasensitive immunochromatographic assay for the simultaneous detection of five chemicals in drinking water, Biosens. Bioelectron. 66 (2015) 445-453, http:// dx.doi.org/10.1016/j.bios.2014.12.004.

[11] C. Sicard, C. Glen, B. Aubie, D. Wallace, S. Jahanshahi-Anbuhi, K. Pennings, G.T Daigger, R. Pelton, J.D. Brennan, C.D.M. Filipe, Tools for water quality monitoring and mapping using paper-based sensors and cell phones, Water Res. 70 (2015) 360-369, http://dx.doi.org/10.1016/j.watres.2014.12.005.

[12] T.G. Aw, J.B. Rose, Detection of pathogens in water: from phylochips to qPCR to pyrosequencing, Curr. Opin. Biotechnol. 23 (2012) 422-430, http://dx.doi. org/10.1016/j.copbio.2011.11.016.

[13] G. Zanchetta, R. Lanfranco, F. Giavazzi, T. Bellini, M. Buscaglia, Emerging applications of label-free optical biosensors, Nanophotonics 6 (2017) 627-645, http://dx.doi.org/10.1515/nanoph-2016-0158.

[14] M. Czugala, C. Fay, N.E. O'Connor, B. Corcoran, F. Benito-Lopez, D. Diamond, Portable integrated microfluidic analytical platform for the monitoring and detection of nitrite, Talanta 116 (2013) 997-1004, http://dx.doi.org/10.1016/j. talanta.2013.07.058. 
[15] M. Li, X. Zhou, W. Ding, S. Guo, N. Wu, Fluorescent aptamer-functionalized graphene oxide biosensor for label-free detection of mercury(II), Biosens. Bioelectron. 41 (2013) 889-893, http://dx.doi.org/10.1016/j.bios.2012.09.060.

[16] F. Giavazzi, M. Salina, R. Cerbino, M. Bassi, D. Prosperi, E. Ceccarello, F. Damin, L. Sola, M. Rusnati, M. Chiari, B. Chini, T. Bellini, M. Buscaglia, Multispot, label-free biodetection at a phantom plastic-water interface, Proc. Natl. Acad. Sci. 110 (2013) 9350-9355, http://dx.doi.org/10.1073/pnas.1214589110.

[17] F. Giavazzi, M. Salina, E. Ceccarello, A. Ilacqua, F. Damin, L. Sola, M. Chiari, B. Chini, R. Cerbino, T. Bellini, M. Buscaglia, A fast and simple label-free immunoassay based on a smartphone, Biosens. Bioelectron. 58 (2014) 395-402, http://dx.doi.org/10.1016/j.bios.2014.02.077.

[18] D. Prosperi, C. Morasso, F. Mantegazza, M. Buscaglia, L. Hough, T. Bellini, Phantom nanoparticles as probes of biomolecular interactions, Small 2 (2006) 1060-1067, http://dx.doi.org/10.1002/smll.200600106.

[19] R. Lanfranco, F. Giavazzi, M. Salina, G. Tagliabue, E. Di Nicolò, T. Bellini, M. Buscaglia, Selective adsorption on fluorinated plastic enables the optical detection of molecular pollutants in water, Phys. Rev. Appl. 5 (2016) 54012, http://dx.doi.org/10.1103/PhysRevApplied.5.054012.

[20] K. Yang, H. Peretz-Soroka, Y. Liu, F. Lin, Novel developments in mobile sensing based on the integration of microfluidic devices and smartphones, Lab Chip. 16 (2016) 943-958, http://dx.doi.org/10.1039/C5LC01524C.

[21] J.C. Jokerst, J.M. Emory, C.S. Henry, Advances in microfluidics for environmental analysis, Analyst 137 (2012) 24-34, http://dx.doi.org/10.1039/ C1AN15368D.

[22] K.F. Lei, Chapter 1. Materials and fabrication techniques for nano- and microfluidic devices, in: Microfluid Detect. Sci., Royal Society of Chemistry, Cambridge, 2014, pp. 1-28, http://dx.doi.org/10.1039/978184973760900001.

[23] R.P. Gandhiraman, C. Volcke, V. Gubala, C. Doyle, L. Basabe-Desmonts, C. Dotzler, M.F. Toney, M. Iacono, R.I. Nooney, S. Daniels, B. James, D.E. Williams, High efficiency amine functionalization of cycloolefin polymer surfaces for biodiagnostics, J. Mater. Chem. 20 (2010) 4116, http://dx.doi.org/10.1039/ b925737c.

[24] J. Saez, J. Etxebarria, M. Antoñana-Diez, F. Benito-Lopez, On-demand generation and removal of alginate biocompatible microvalves for flow control in microfluidics, Sens. Actuators B Chem. 234 (2016) 1-7, http://dx. doi.org/10.1016/j.snb.2016.04.140.

[25] R. Byrne, F. Benito-Lopez, D. Diamond, Materials science and the sensor revolution, Mater. Today 13 (2010) 16-23, http://dx.doi.org/10.1016/S13697021(10)70124-3.

[26] M. Czugala, R. Gorkin III, T. Phelan, J. Gaughran, V.F. Curto, J. Ducrée, D. Diamond, F. Benito-Lopez, Optical sensing system based on wireless paired emitter detector diode device and ionogels for lab-on-a-disc water quality analysis, Lab Chip. 12 (2012) 5069, http://dx.doi.org/10.1039/c2lc40781g.

[27] No Title, (n.d.). http://www.solvay.com/en/markets-and-products/featuredproducts/hyflon-ad.html.

[28] F. Tasselli, Non-solvent induced phase separation process (NIPS) for membrane preparation, in: Encycl. Membr, Springer Berlin Heidelberg, Berlin, Heidelberg, 2014, pp. 1-3, http://dx.doi.org/10.1007/978-3-642-40872-4_ 1823-1.

[29] J. Saez, L. Basabe-Desmonts, F. Benito-Lopez, Low-cost origami fabrication of 3D self-aligned hybrid microfluidic structures, Microfluid. Nanofluid. 20 (2016) 116, http://dx.doi.org/10.1007/s10404-016-1781-7.

[30] J. Elizalde, M. Antoñana, L. Matthys, F. Laouenan, J.M. Ruano-López, Rapid prototyping of self aligned 3D microfluidic structures, 17th Int. Conf. Miniaturized Syst. Chem. Life Sci. MicroTAS 2013 (2013).

[31] H.C. vandeHulst, Light Scattering by Small Particles, Dover, New York, 1957, https://books.google.it/books/about/Light_Scattering_by_Small_Particles. html?id=PlHfPMVAFRcC\&pgis=1 (Accessed 11 August 2015).
[32] R. Piazza, V. Degiorgio, Light scattering study of spherical latex particles: measurement of surfactant adsorption and of intrinsic anisotropy, Opt. Commun. 92 (1992) 45-49, http://dx.doi.org/10.1016/0030-4018(92)90216E.

[33] I. Langmuir, The adsorption of gases on plane surfaces of glass, mica and platinum, J. Am. Chem. Soc. 40 (1918) 1361-1403, http://dx.doi.org/10.1021/ ja02242a004.

[34] B. Zhao, C.W. MacMinn, R. Juanes, Wettability control on multiphase flow in patterned microfluidics, Proc. Natl. Acad. Sci. 113 (2016) 10251-10256, http://dx.doi.org/10.1073/pnas.1603387113. 